\title{
DDS Elementary Style: A Brief Guide for Authors
}

\author{
Jonathan D. Kaunitz ${ }^{1}$
}

Published online: 16 June 2016

(C) Springer Science+Business Media New York (Outside the USA) 2016

Medical writing entails a vocabulary and idiomatic style rarely used elsewhere. Integrating the shorthand, abbreviations, colloquialisms, and initialisms used in clinical care with the technical terms and phraseology intrinsic to the field, medical writing has the potential to be stilted, wordy, and confusing. As Editor-in-Chief of Digestive Diseases and Sciences, I am responsible for not only the published content but also the form and style in which the content is written. I have taken this responsibility literally for all of the editorials, much of the content of the special sections, and all of the DDS Distinguished Digestive Seminars published, which are edited for English usage, grammar, syntax, and style in addition to content. Although somewhat time consuming, this exercise not only helps me stay abreast of the journal's content, but also has enabled the journal to cultivate a distinctive style that I hope will be pleasing and helpful to our readership.

One could ask: "Why go to this time and trouble-is not most of what is written good enough?" The answer is that our entire authorship is highly educated, well read, and experienced. During the years devoted to obtaining an education and afterward, although emphasis is placed on content, little appears to be placed on style, grammar, or syntax. As a consequence, most medical writing, although technically sound and scientifically accurate, is often rife with jargon, clichés, awkward phraseology, and any number of English misadventures that detract from its impact, readability, and forcefulness of the content. Another

Jonathan D. Kaunitz

jake@ucla.edu

1 Departments of Medicine and Surgery, Greater Los Angeles VA Healthcare System, UCLA School of Medicine, Los Angeles, CA 90073, USA

consideration is that centuries hence, publications will be universally and instantly accessible rather than moldering forgotten in the basement of a medical library, adding incentive to write in an elegant, concise, and vigorous style, if not for now, for posterity. The purpose of this editorial is to alert our authors to the many common grammatical phrases and words commonly used in scientific writing that could be improved by adhering to a few simple concepts, which should improve the readability and impact of the publication. Although one could argue that in medical writing, style is superfluous, to me a well-written article not only reinforces the author's reputation as a writer, but also as a scientist.

The inspiration for this piece is the ageless, pithy, and slim volume entitled The Elements of Style originally authored by Strunk Jr. [1] and expanded by White [2]. Nearly a century later, the book's core themes and advice, stressing the use of the minimum number of well-chosen words necessary to convey a point or idea, still hold true. What I would like to do here is to similarly highlight recurring phrases and usage unique to or at least typical of medical writing, the clarity of which can be substantially improved with minimal modification.

The following phrases, commonly used in medical writing, likely to have been uncritically absorbed by younger generations encountering them in the medical literature, are worthy of modification:

results in an increase (decrease) of

have been shown to be increased

acts as a mobilizer of

works by mobilizing the recruitment of

in the range of $200-400 \mathrm{pg} / \mathrm{ml}$
Increases (decreases)

is (are) increased

mobilizes

recruits

200-400 pg/ml 
In every case illustrated above, a 5- to 6-word phrase was replaced by a well-chosen verb that not only deletes and replaces excess words, but conveys the author's intent more directly and forcefully. The same principles apply to any phrase in which another verb is used as a noun requiring the use of a non-specific verb:

\begin{tabular}{ll}
\hline do surgery & operate \\
have been used to facilitate & have facilitated \\
placed on anti-tuberculous therapy & treated for \\
& tuberculosis
\end{tabular}

These expressions also commonly crop up, often to the author's detriment:

\begin{tabular}{ll}
\hline it has also been suggested that & [delete] \\
it has been demonstrated that & [delete] \\
it has been shown to be & [delete] \\
it is interesting to note that & [delete] \\
new studies have demonstrated that & [delete] \\
in the setting of & [delete] \\
also worth considering is the fact that & [delete] \\
\hline
\end{tabular}

Deletion of these mostly needless, wordy, and gratuitous phrases that are already implicit by context universally improves readability by focusing the reader's attention on the key elements of the publication, whether it be the data or the authors' principal arguments or conclusions. As an example, if you are describing someone's data, it is not necessary to introduce it by using the phrase "it has been shown that" since it should be obvious from the context what you are describing.

"On" is a preposition meaning "atop," "arranged," "continuous," and "scheduled," among other meanings. It is frequent used clinically in expressions such as:

\begin{tabular}{ll}
\hline on radiological studies & radiologically \\
on examination & examination revealed \\
the patient was on steroids & corticosteroids were administered \\
$\begin{array}{l}\text { was not evident on initial } \\
\text { review }\end{array}$ & $\begin{array}{c}\text { was not evident when reviewed } \\
\text { initially }\end{array}$ \\
\hline
\end{tabular}

In every case illustrated above, the non-specific preposition is either replaced by a specific verb or the phrase can be shortened by the use of a single adverb, conveying the meaning more directly and concisely.

Sentences that are connected by "and" can usually be rewritten to start with "even though," "although," or a similar concessive clause, eliminating the need for the conjunction "and" while improving meaning and readability.

\begin{tabular}{lc}
\hline $\begin{array}{c}\text { The liver lesions were small, } \\
\text { and they were easy to } \\
\text { visualize radiographically... }\end{array}$ & $\begin{array}{c}\text { Although the liver lesions were } \\
\text { small, they were easy to } \\
\text { visualize radiographically... }\end{array}$ \\
\hline
\end{tabular}

In general, two phrases connected with "and" are usually part of a run-on sentence, a name derived from its awkward and stilted construction that sounds fragmented. Starting with "although" or the like alerts the reader that the second phrase logically follows the first, obviating the need for multiple reads to fully comprehend the meaning.

Another word not always used correctly is:

both (used to introduce a list of two items)

[delete]

This is where I diverge from Mssrs. Strunk and White, since the use of "both" to introduce a list of two items, e.g., "...both infliximab and adalimumab are biological agents..." is gratuitous, and this can safely be deleted.

Another word that often misused in medical writing is "known" which is usually gratuitous since the statement in which it is used is usually stating data or other "known" facts:

are known to have malignant potential are potentially malignant

Another needless phrase:

Further studies are needed to...

[delete]

This sentence usually closes an editorial or other work. Although it can be useful if the "further studies" are well thought out and non-obvious, it usually adds little to include this phrase if the reader already knows what you are going to say. I am sure every scientist extant is aware that more research needs to be done about every imaginable topic. Why state what is already evident?

I will now highlight single words and short phrases frequently used in medical writing that are commonly used incorrectly. Please note that although the opinions stated and recommendations provided are personal, highly subjective, and counter to the writing style used in the bulk of medical writing, they do represent incorrect usage of common English words, reflecting my somewhat fussy approach to writing, in which precision trumps customary 
practice. Nevertheless, correct word usage informs the reader that you are meticulous and a stickler for details, never a bad impression to give in science:

level(s)

concentration(s)

I am not sure how the term "level" entered scientific discourse to replace the correct term "concentration." I am sure, however, that it should not be used in formal scientific writing.

significant

substantial (if not statistically significant)

In medical writing, where statistical tests are routinely used for data comparisons, the term "significant" should only refer to statistical comparisons, and should not be used to indicate a large effect outside of statistical contexts:

media medium

"Media" is the plural of "medium" and should not be used when the singular form is intended, which is most of the time. Not to be confused with "the media" referring to means of mass communication such as newspapers, the Internet, and radio.

allow

enable, facilitate

"Allow" is usually meant as tolerate, accept, or acknowledge. It is often used in scientific writing thus: "Antiviral drugs allow clinicians to effectively treat hepatitis." In this context, "enable," which usually means empower or facilitate, is generally more appropriate.

Another commonly misused adjective:

"Critical" means serious, dangerous, or life threatening as in "critical care." It can mean judgmental or essential, but should not be used as a modifier, i.e., "critically important" or when a large effect is meant, where "sizable," "considerable," and "important" are more appropriate.
Another commonly misused word:

found (to have)

localized, expressed, reported, (had)

"Found" is often used thus: "...isomaltase is found in the intestinal brush border..." when "expressed or localized" would be far more direct and accurate. Another common misuse, "...Jones et al. found that mesalamine...". When used to describe an author's findings, "found" is better replaced by "reported."

Another misused word is "seen":

\begin{tabular}{|c|c|}
\hline no improvement was seen & no improvement was observed \\
\hline $\begin{array}{l}\text { focal lesions seen on } \\
\text { ultrasound }\end{array}$ & $\begin{array}{l}\text { Focal lesions reported by the } \\
\text { ultrasonographer }\end{array}$ \\
\hline
\end{tabular}

Another word frequently used in the clinical record:

presented
(with)
complained of, was initially manifest

This is a rare instance of a single word replaced by a longer and somewhat awkward-sounding phrase. Although brevity and concision are desirable, the misuse of words is not. "Present" can be used as a noun or an adjective usually defined as current, contemporary, or existent. The verb "presented" is usually defined as gave, handed over, offered, or furnished with.

Another term commonly used in clinical medicine, in particular among gastroenterologists, is:

melanotic or melenic stool

melena

Melanotic, meaning containing melanin, would seldom apply to stool, and melenic is a made-up. Why not just use the accepted (and rather melodious sounding) noun "melena?"

Another example of a commonly used word that has no place in medical writing:

\begin{tabular}{ll}
\hline played a role in & $\begin{array}{c}\text { contributed to, was a component of, } \\
\text { influenced }\end{array}$ \\
$\begin{array}{l}\text { no evidence of its role in } \\
\text { enhancing }\end{array}$ & $\begin{array}{l}\text { no evidence supports its enhancement } \\
\text { of }\end{array}$ \\
\hline
\end{tabular}


The word "role" was imported to English from the French in 1875, formerly spelled in the French manner: rôle. The term, although widely used to describe a function or contribution of something to a larger whole, should be reserved for theatrical contexts, although in medicine it has been adopted by sociologists who use it correctly to describe an individual's expected or characteristic social behavior.

A commonly misused adjective:

dramatic

substantial, sizable

Borrowing another term from our thespian colleagues, "dramatic" is often used to mean a considerable, sizable, or important effect or contribution, rather than its actual meaning of histrionic, theatrical, or stagy.

Again, I emphasize that since these comments are personal, they should not be considered as absolute or binding, in particular since I am sure that there are many whose medical English usage is far superior to mine. Nevertheless, since these simple rules of usage have served well over the past few years and several hundred publications, I urge our authors to always consider if each word or phrase is necessary, and if not, whether it can be deleted or replaced by a word, phrase, or grammatical construction that conveys the same meaning more concisely and directly.

Also worth considering is the fact that further studies are necessary to Thank you for your support of and for your past and continued contributions to Digestive Diseases and Sciences.

Acknowledgments I would like to thank my Managing Editor, Meghan Keeffe, for her helpful suggestions, careful edits, and unfailing efforts to improve the content of Digestive Diseases and Sciences.

\section{References}

1. Strunk, W. Elements of style. Ithaca, NY: Priv. Print (Geneva, NY: Press of W. F. Humphrey); 1918.

2. Strunk W, White EB. The elements of style. New York: Macmillan; 1959. 